\title{
Non-repetitive 3-coloring of subdivided graphs
}

\author{
Andrzej Pezarski \\ Theoretical Computer Science Department \\ Faculty of Mathematics and Computer \\ Science, Jagiellonian University \\ Krakow, Poland \\ pezarski@tcs.uj.edu.pl
}

\author{
Michał Zmarz \\ Theoretical Computer Science Department \\ Faculty of Mathematics and Computer \\ Science, Jagiellonian University \\ Krakow, Poland \\ mrozik@tcs.uj.edu.pl
}

Submitted: Dec 22, 2008; Accepted: May 12, 2009; Published: May 20, 2009

Mathematics Subject Classification: 05C15

\begin{abstract}
We show that every graph can be subdivided in a way that the resulting graph can be colored without repetitions on paths using only 3 colors. This extends the result of Thue asserting the existence of arbitrarily long nonrepetitive strings over a 3-letter alphabet.
\end{abstract}

\section{Introduction}

A vertex coloring $f$ of an undirected graph $G$ is nonrepetitive if there is no simple path $v_{1}, \ldots, v_{2 r}$ in $G$ such that $f\left(v_{i}\right)=f\left(v_{r+i}\right)$ for all $i=1, \ldots, r$. The minimum number of colors needed in such coloring is called Thue chromatic number and is denoted by $\pi(G)$. Of course, every nonrepetitive coloring is also a proper coloring, so $\chi(G) \leqslant \pi(G)$.

A subdivision of an undirected graph $G$ is a graph resulting from a (possibly empty) sequence of the edge subdivisions applied to $G$. A subdivision of an edge $e=\{u, v\}$ of the graph $G$ is obtained by the addition of a new vertex $w$ into $G$ and replacement of $e$ with two new edges $\{u, w\}$ and $\{w, v\}$.

For a graph $G$ we want to find

$$
\pi_{S}(G)=\min \{\pi(F): F \text { is a subdivision of } G\}
$$

and to effectively determine a subdivision of $G$ that witnesses $\pi_{S}(G)$.

In 1906 Axel Thue published his paper [Thu06] in which he presented an infinite, square-free sequence of digits $0,1,2$, that is in which no subword is repeated twice in a row. This can be expressed as $\pi(\mathcal{P}) \leqslant 3$ for every path $\mathcal{P}$. It implies easily that $\pi\left(\mathcal{C}_{n}\right) \leqslant 4$ for every cycle $\mathcal{C}_{n}$. Recently Currie proved [Cur02] that $\pi\left(\mathcal{C}_{n}\right)=3$ for every $n \geqslant 18$. By combining these two results we obtain $\pi_{S}(G) \leqslant 3$ for every graph $G$ with 
maximum degree at most 2 . It is also known that $\pi(T) \leqslant 4$ for every tree $T$, see [BGK $\left.{ }^{+} 07\right]$. Moreover $\pi_{S}(T) \leqslant 3$, as proved in $\left[\mathrm{BGK}^{+} 07\right]$.

An effort has been made to bound $\pi_{S}(G)$ without restricting $G$ to any specific class of graphs. In fact, it is easy to show that $\pi_{S}(G) \leqslant 5$ for every graph $G$ [Gry07]. This bound was improved to 4 by Barát and Wood [BW08] and independently by Marx and Shaefer [MS09].

In fact, Grytczuk [Gry07] and Barát and Wood [BW08] conjectured that $\pi_{S}(G) \leqslant 3$ for every graph $G$. The aim of this paper is to confirm this conjecture. Our proof is based on the original construction of Thue and is similar to the approach by Marx and Schaefer. We give it in Section 3 after a necessary preparation in Section 2.

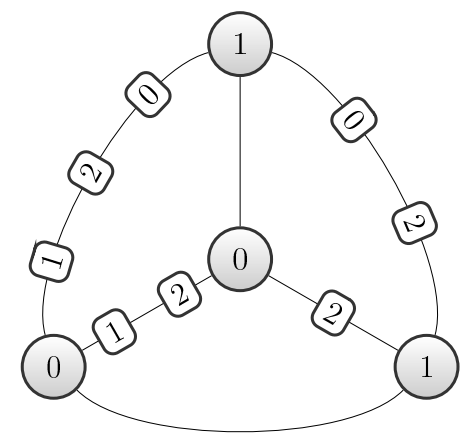

Figure 1: Non-repetitive 3-coloring of a subdivided clique $\mathcal{K}_{4}$

For other interesting problems on Thue colorings of graphs, see [AGHR02].

\section{Preliminaries}

First, we introduce some notation that will be used later. Upper left arrow denotes reversion of a string, that is

$$
\overleftarrow{a_{1} a_{2} \ldots a_{n}}=a_{n} a_{n-1} \ldots a_{1} .
$$

Upper bar denotes complement modulo 2, that is

$$
\overline{a_{1} a_{2} \ldots a_{n}}=\left(2-a_{1}\right)\left(2-a_{2}\right) \ldots\left(2-a_{n}\right),
$$

where $a_{i} \in\{0,1,2\}$. Square brackets are used to extract the substring from a string, i.e., $a_{1} a_{2} \ldots a_{n}[i]=a_{i}$ and $a_{1} a_{2} \ldots a_{n}[i \ldots j]=a_{i} \ldots a_{j}$.

The sequence $\gamma=\left(\gamma_{0}, \gamma_{1}, \ldots\right)$ of Thue-Morse binary strings is defined as follows:

$$
\gamma_{0}=0
$$

and for $i>0, \gamma_{i}$ arises from $\gamma_{i-1}$ by applying the substitutions

$$
0 \rightarrow 01 \quad 1 \rightarrow 10
$$


to all digits in $\gamma_{i-1}$. The first few strings $\gamma_{i}$ are listed in Table 1.

By counting the number of 1's between consecutive 0's in $\gamma_{i}$ we obtain consecutive digits of a new string, which we denote $\beta_{i}$. More formally, if $c_{i}^{j}$ is the position in $\gamma_{i}$ at which the $j$-th 0 occurs then $\beta_{i}[j]=c_{i}^{j+1}-c_{i}^{j}-1$.

The strings $\beta_{i}$ were first defined by Thue [Thu06] who showed that each $\beta_{i}$ is nonrepetitive and consist only of digits $0,1,2$. As before, the first few values of $\beta_{i}$ are listed in Table 1.

\begin{tabular}{l|l|l}
\multicolumn{3}{|c}{ Table 1: $\gamma$ and $\beta$ sequences } \\
$i$ & $\gamma_{i}$ & $\beta_{i}$ \\
\hline 0 & 0 & \\
1 & 01 & 2 \\
2 & 0110 & 210 \\
3 & 01101001 & 2102012 \\
4 & 0110100110010110 & 210201210120210 \\
5 & 01101001100101101001011001101001
\end{tabular}

Lemma 2.1. No $\beta_{i}$ contains 010 or 212 as a substring.

Proof. For 010 to appear as a substring of some $\beta_{i}, 00100$ must be a subword of $\gamma_{i}$. As $\gamma_{i}$ is constructed from $\gamma_{i-1}$ by substituting a single digit by a pair of different digits, there is no possibility that two leading and simultaniously two last zeros in 00100 can occur.

Analogously, 212 in $\beta_{i}$ gives rise to 011010110 in $\gamma_{i}$ which cannot happen by the very same token applied to the first two and the last two 1's.

Lemma 2.2. For every natural number $m$, there is a family of strings $\left\{\alpha_{i}: i=1,2, \ldots, m\right\}$ over $\{0,1,2\}$ satisfying the following properties:

1. $\alpha_{i} \neq \alpha_{j}$, for $i \neq j$

2. $\alpha_{i} \neq \overleftarrow{\alpha_{j}}$, for all $i, j$

3. every string $\alpha_{i}$ is nonrepetitive

4. all strings have the same length, i.e. $\left|\alpha_{i}\right|=\left|\alpha_{j}\right|$ and moreover $\left|\alpha_{j}\right|=O(m)$

5. all strings begin with 1020 and end with 2021 but no string contains 010 nor 212 as a subword.

Proof. Recall, that

$$
\beta_{5}=210201210120210, \quad \overline{\beta_{5}}=012021012102012 .
$$


Observe, that

$$
\begin{aligned}
& \beta_{6}=\beta_{5} \cdot 2 \cdot \overline{\beta_{5}} \\
& \beta_{7}=\beta_{5} \cdot 2 \cdot \overline{\beta_{5}} \cdot 1 \cdot \overline{\beta_{5}} \cdot 0 \cdot \beta_{5} \\
& \beta_{8}=\beta_{5} \cdot 2 \cdot \overline{\beta_{5}} \cdot 1 \cdot \overline{\beta_{5}} \cdot 0 \cdot \beta_{5} \cdot 2 \cdot \overline{\beta_{5}} \cdot 0 \cdot \beta_{5} \cdot 1 \cdot \beta_{5} \cdot 2 \cdot \overline{\beta_{5}}
\end{aligned}
$$

and more generally, for $i \geqslant 2$ we have

$$
\beta_{i}= \begin{cases}\beta_{i-1} \cdot 2 \cdot \overline{\beta_{i-1}}, & \text { if } i \text { is even } \\ \beta_{i-1} \cdot 1 \cdot \overline{\beta_{i-1}}, & \text { if } i \text { is odd }\end{cases}
$$

Let $k=6+\left\lceil\log _{2} m\right\rceil$. The string $\beta_{k}$ contains at least $m$ (disjoint) instances of $\beta_{5}$ as subwords. Now, let $p_{i}$ denote the position of the $i$-th occurrence of $\beta_{5}$ in $\beta_{k}$, which means that $\beta_{k}\left[p_{i} . . p_{i}+14\right]=\beta_{5}$.

We know that $\beta_{k+2}$ has length $\left|\beta_{k+2}\right|=4\left|\beta_{k}\right|+3$ and contains two instances of $\beta_{k}$ shifted by $d=3\left|\beta_{k}\right|+3$ positions. In particular, for $i=1,2, \ldots, m$ we have

$$
\beta_{k+2}\left[p_{i}+d . . p_{i}+14+d\right]=\beta_{k+2}\left[p_{i} . . p_{i}+14\right]=\beta_{5}
$$

for $i=1,2, \ldots, m$.

Finally, we define our strings by

$$
\alpha_{i}=\beta_{k+2}\left[p_{i}+1 . . p_{i}+13+d\right] \quad i=1,2, \ldots, m
$$

Clearly, this definition satisfies $(3)-(5)$. To see (1), assume to the contrary that $\alpha_{i}=\alpha_{j}$ for some $i<j$. Since the $\alpha$-strings have length $d+13>\frac{\left|\beta_{k+2}\right|}{2}$, any two of them have to overlap so that this overlap is realized by

$$
\alpha_{i}=u \cdot v \quad \alpha_{j}=v \cdot w,
$$

where $u \cdot v \cdot w$ is a substring of $\beta_{k+2}$. But

$$
u \cdot v \cdot w=u \cdot \alpha_{j}=u \cdot \alpha_{i}=u \cdot u \cdot v
$$

has a repetition, which cannot appear in $\beta_{k+2}$.

Finally, (2) follows directly from (5).

\section{Main result}

Theorem 3.1. Every graph has a subdivision that can be nonrepetitively colored using 3 colors.

Proof. For a graph $G=(V, E)$, let $m=2|E|$ and $\alpha_{1}, \alpha_{2}, \ldots, \alpha_{m}$ be the $\alpha$-strings satisfying the conditions of Lemma 2.2. Subdivide every edge of $G$ by placing $2\left|\alpha_{i}\right|+9$ new vertices on it and denote the resulting graph by $G^{\prime}=\left(V^{\prime}, E^{\prime}\right)$. 
Now we color $G^{\prime}$. Assign the color 1 to all vertices from $V$. Color newly added vertices on the $i$-th edge from $E$ in such a way that reading them in order of their placement gives a string

$$
e_{i}=20 \cdot \alpha_{2 i-1} \cdot 20102 \cdot \overleftarrow{\alpha_{2 i}} \cdot 02
$$

The numbering and orientation of edges from $E$ does not matter.

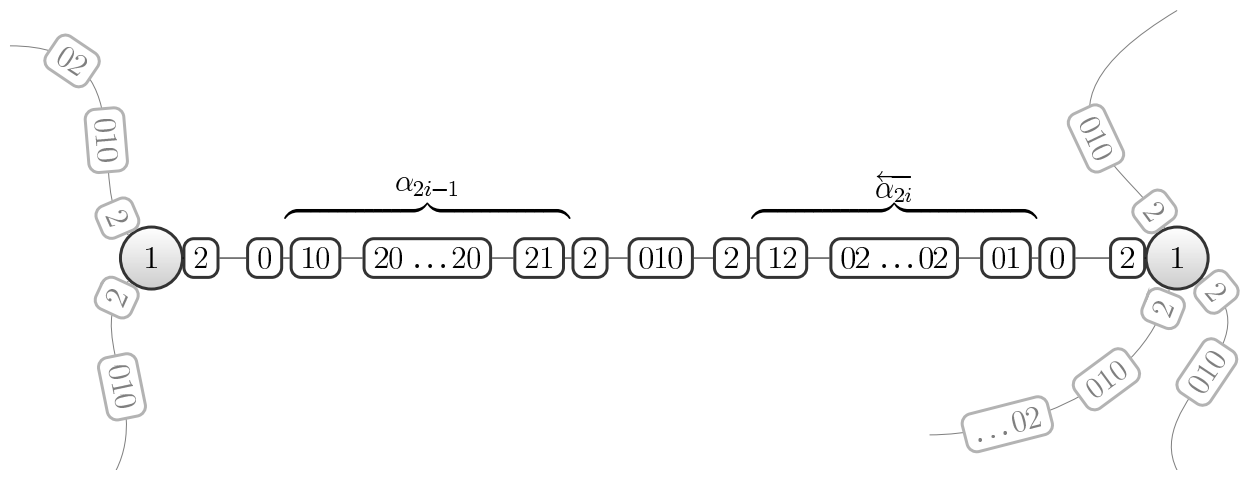

Figure 2: Coloring of the subdivision

Take any simple path $\mathcal{P}$ from the subdivided graph $G^{\prime}$ and let $P$ be the word consisting the of the labels of the vertices on $\mathcal{P}$. Our aim is to show that $P$ cannot be of the form $P=w \cdot w$ for any word $w$.

The string $P$ may contain one or more occurences of 010 or 212. Those substrings of $P$ will play a crucial role in our proof by serving as tags. By Lemma 2.2.(5) $\alpha$-strings contain no tags. Therefore, every two consecutive occurences of tags in $P$ are either adjacent to each other or are separated by an occurence of $\alpha_{i}\left[3 . .\left|\alpha_{i}\right|-2\right]$ or $\overleftarrow{\alpha_{i}\left[3 . .\left|\alpha_{i}\right|-2\right]}$, for some $i$.

Recall that every $\alpha_{i}$ was used only once to color vertices from $G^{\prime}$ and that $\mathcal{P}$ is a simple path. This, together with Lemma 2.2.(5) leads to the following conclusion:

Fact 3.2. Every $\alpha_{i}$ may occur at most once as a subword in $P$ or in $\overleftarrow{P}$

Now we consider how long $P=w \cdot w$ might be. If $\mathcal{P}$ contains at least two vertices from $V$ then $P$ has to contain (at least) two instances of some $\alpha$-strings, with one of them being reversed. One of those two instances must be located entirely in $w$, so that for some $i$ the word $P=w \cdot w$ contains $\alpha_{i}$ twice or $\overleftarrow{\alpha_{i}}$ twice. In particular, $\mathcal{P}$ contains two different fragments colored by $\alpha_{i}$ (or $\overleftarrow{\alpha_{i}}$ ), contrary to Fact 3.2. Therefore $\mathcal{P}$ contains at most one vertex from $V$.

Hence $P$ has to be a subword of the word:

$$
M=20 \cdot \alpha_{a} \cdot 20102 \cdot \overleftarrow{\alpha_{b}} \cdot 02120 \cdot \alpha_{c} \cdot 20102 \cdot \overleftarrow{\alpha_{d}} \cdot 02
$$

that results from painting two consecutive edges from $E$ by $20 \cdot \alpha_{a} \cdot 20102 \cdot \overleftarrow{\alpha_{b}} \cdot 02$ and $20 \cdot \alpha_{c} \cdot 20102 \cdot \overleftarrow{\alpha_{d}} \cdot 02$ with $|a-b|=1$ and $|c-d|=1$. Looking more closely how $\alpha$-strings start and end we see that 


$$
\begin{gathered}
M=2 \cdot 0 \overbrace{10 \cdot 20 \cdot \ldots \cdot 20 \cdot 21}^{\overbrace{a}^{\alpha_{a}}} 2 \cdot 010 \cdot 2 \overbrace{12 \cdot 02 \cdot \ldots \cdot 02 \cdot 01}^{\overbrace{\alpha_{b}}} 0 . \\
212 \cdot \underbrace{10 \cdot 20 \cdot \ldots \cdot 20 \cdot 21}_{\alpha_{c}} 2 \cdot 010 \cdot 2 \underbrace{12 \cdot 02 \cdot \ldots \cdot 02 \cdot 01}_{\overleftarrow{\alpha_{d}}} 0 \cdot 2 .
\end{gathered}
$$

The rest of the argument splits into cases depending on the number of tags appearing in $P$.

If $P=w \cdot w$ does not contain any tag then it must be entirely contained in one of: 201, $\alpha_{a}, 1201,1021, \overleftarrow{\alpha_{b}}, 1021,1201, \alpha_{c}, 1201,1021, \overleftarrow{\alpha_{d}}, 102$. Since none of them contain a repetition, we are done.

Now suppose that $P$ contains exactly one tag. Of course this tag must start in the first and finish in the second half of $P=w \cdot w$, having something in common with both $w$ 's in $P$. There are no tags in $\alpha$-strings and each tag from $M$ is adjacent to some other tag, with the exception of the first tag that occurs very closely (at distance 1 ) to the beginning of $M$ and the last tag that occurs very closely to the end of $M$. Therefore, $P$ cannot be too long, namely $|P| \leqslant 8$. By inspecting possible positions of our tag in $P$ and how $P$ lies within $M$, we are assured that $P$ cannot be a repetition. For this inspection we simply analyze the digits presented in $(*)$.

Finally, in the rest of the proof we assume that there are at least two tags in $P$. To analyze how the tags can occur in $P$ we fix their positions in $M$, which are simply defined to be the positions of their first digits. Let $s=\left|\alpha_{a}\right|=\left|\alpha_{b}\right|=\left|\alpha_{c}\right|=\left|\alpha_{d}\right|$. Note that $M$ contains 11 tags and their positions are: $t_{1}=2, t_{2}=s+1, t_{3}=s+4, t_{4}=s+7$, $t_{5}=2 s+6, t_{6}=2 s+9, t_{7}=2 s+12, t_{8}=3 s+11, t_{9}=3 s+14, t_{10}=3 s+17$, $t_{11}=4 s+16$. Note that for odd $i, t_{i}$ denotes a position of a 010 tag, while $t_{i}$ with even $i$ denotes a position of a $212 \mathrm{tag}$.

Since $P=w \cdot w$ contains at least two tags, we know that at least one of them is fully contained in $w$. We focus on the first $\operatorname{tag} T$ that occurs in $P$, so that after embedding $P$ into $M$ we know that $M\left[t_{i} . . t_{i}+2\right]=T$ and $M\left[t_{i}+|w| . . t_{i}+|w|+2\right]=T$, for some $i$. The minimum distance between tags in $M$ of the same type is 6 , therefore $|w| \geqslant 6$.

Suppose $|w|=6$. There are 3 pairs of tags in $M$ that are of the same type and in each pair tags are placed at distance 6 . Their positions are: $\left(t_{2}, t_{4}\right),\left(t_{5}, t_{7}\right),\left(t_{8}, t_{10}\right)$, so that for some $i \in\{2,5,8\}, w \cdot w$ contains $M\left[t_{i} . . t_{i+2}+2\right]$ and at least two additional digits $M\left[t_{i}-2 . . t_{i}-1\right]$ or $M\left[t_{i+2}+3 . . t_{i+2}+4\right]$. However these two additional digits do not match digits in the middle tag $M\left[t_{i} . . t_{i+1}+2\right]$. Again, it is sufficient to analyze only digits presented in $(*)$.

Hence $|w|>6$ and referring to the structure of $M$ we observe that the length of $w$ must be even greater than $s$. Since $|w|>s>12, w$ has to contain at least 5 digits either to the left or to the right of tag $T$. Again by inspecting $M$ described by $(*)$ we see that the only pair of matching tags that occur in the first and the second $w$ of $P=w \cdot w$ consist of tags $M\left[t_{i} . . t_{i}+2\right]$ and $M\left[t_{i+6} . . t_{i+6}+2\right]$. For each such pair $|w|=2 s+10$, so that $|P|=4 s+20$. But this is more than $|M|=4 s+19$. This means that $P$ cannot be nested inside $M$ and ends the proof. 


\section{Conclusion}

It is easy to see that if a graph contains $\mathcal{P}_{4}$ - a path consisting of four vertices, then it cannot be nonrepetitively colored by 2 colors. It is also obvious that every graph that contains a cycle requires at least 3 colors in such coloring.

This leads to the formula that gives the exact value of $\pi_{S}(G)$ for any undirected graph $G=(V, E)$

$$
\pi_{S}(G)= \begin{cases}1, & \text { if } E=\emptyset \\ 2, & \text { if } E \neq \emptyset \text { and } G \text { is a star forest } \\ 3, & \text { otherwise. }\end{cases}
$$

By a star forest we mean any acyclic graph that does not contain $\mathcal{P}_{4}$.

\section{Acknowledgement}

We would like to thank Jarosław Grytczuk and Paweł Idziak for their useful comments, suggestions and help in writing this article.

\section{References}

[AGHR02] Noga Alon, Jarosław Grytczuk, Mariusz Hałuszczak, and Oliver Riordan. Nonrepetitive colorings of graphs. Random Struct. Algorithms, 21(3-4):336-346, 2002.

[BGK $\left.{ }^{+} 07\right]$ B. Brešar, J. Grytczuk, S. Klavžar, S. Niwczyk, and I. Peterin. Nonrepetitive colorings of trees. Discrete Mathematics, 307(2):163 - 172, 2007.

[BW08] János Barát and David R. Wood. Notes on Nonrepetitive Graph Colouring. Electron. J. Combin., 15(1):\#R99, 2008.

[Cur02] James D. Currie. There are ternary circular square-free words of length $n$ for $n \geq 18$. Electron. J. Combin., 9(1):\#N10, 2002.

[Gry07] Jarosław Grytczuk. Nonrepetitive colorings of graphs - a survey. Int. J. Math. Math. Sci., 2007:74639, 2007.

[MS09] Dániel Marx and Marcus Schaefer. The Complexity of Nonrepetitive Coloring. Discrete Applied Mathematics, 157(1):13 - 18, 2009.

[Thu06] Axel Thue. Über unendliche Zeichenreihen. Norske Vid. Selsk. Skr. I. Mat. Nat. Kl. Christiana 1 - 22, 1906. 